\title{
Modulation de l'expression des gènes par l'insuline
}

Les effets métaboliques de l'insuline sont multiples; certains passent par la modification de l'expression de gènes, qui peuvent être activés ou réprimés. Les niveaux du contrôle par l'insuline peuvent être transcriptionnels et post-transcriptionnels. Rien n'est encore pratiquement connu des seconds messagers de l'action de l'insuline sur l'expression des gènes ni des éléments d'ADN (effets transcriptionnels) ou d'ARN (effets post-transcriptionnels) qui confèrent la réponse à l'hormone.

\section{Christian Dani \\ Chargé de recherche à l'Inserm}

\section{Paul Grimaldi}

Chargé de recherche à l'Inserm

\section{RÉFÉRENCES}

1. Horvat A. Stimulation of RNA synthesis in isolated nuclei by an insulin-induced factor in liver. Nature 1980; 286 : 906-8.

2. Pilkis SJ, Park CR. Mechanism of action of insulin. Ann Reu Pharmacol 1974 ; 14 : 36885.

\section{ADRESSE}

C. Dani, P. Grimaldi : laboratoire de biologie du développement du tissu adipeux, centre de biochimie (Cnrs LP 7300), faculté des Sciences, parc Valrose, 06034 Nice Cedex, es mécanismes de contrôle par l'insuline des fonctions métaboliques des tissus cibles sont complexes et multiples. En effet, outre ses effets rapides sur le transport des nutriments ou sur l'activité de certaines protéines par modification covalente, l'hormone peut exercer son action régulatrice en modulant l'expression de gènes codant pour les enzymes clés de différentes voies métaboliques. Les connaissances du mode d'action de l'insuline sur l'expression des gènes sont récentes et encore très fragmentaires. De nombreux travaux, souvent réalisés chez l'animal diabétique, ont décrit des effets stimulateurs de l'insuline sur la synthèse globale des protéines du tissu adipeux, du foie ou du muscle squelettique. Ces effets globaux, relativement modérés en amplitude, ont été attribués à une action de l'hormone sur la synthèse des ARN [1], et à une action sur la machinerie de la traduction des ARN messagers passant par l'activation de facteurs de traduction [2] ou par la phosphorylation de protéines ribosomiques [3]. Toutefois ces mécanismes globaux ne peuvent être invoqués pour expliquer les effets insuliniques spécifiques et de grande amplitude sur l'expression de certains gènes dans un tissu particulier.

Depuis une dizaine d'années, plusieurs études ont mis en évidence de telles actions spectaculaires de l'insuline sur l'expression de gènes et sur le métabolisme des ARN messagers correspondants ; une liste non exhaustive de ces gènes est présentée dans le Tableau I. Il faut noter que les études réalisées in vivo, en parti- 
Tableau I

GĖNES DONT L'EXPRESSION EST MODULÉE PAR L'INSULINE

\begin{tabular}{|c|c|c|c|}
\hline Gènes & $\begin{array}{c}\text { Tissus ou modèles } \\
\text { cellulaires }\end{array}$ & $\begin{array}{c}\text { Effets de } \\
\text { l'hormone }\end{array}$ & Références \\
\hline \multirow[t]{2}{*}{$\begin{array}{l}\text { Phosphoénolpyruvate } \\
\text { carboxykinase }\end{array}$} & $\begin{array}{l}\text { Foie de rat, cellules } \\
\text { H4IIE }\end{array}$ & Inhibiteur & {$[4,5]$} \\
\hline & $\begin{array}{l}\text { Préadipocytes Ob17 et } \\
\text { 3T3-F442A }\end{array}$ & Stimulateur & [6] \\
\hline Acide gras synthétase & Foie de rat & Stimulateur & [7] \\
\hline Pyruvate kinase & Foie de rat & Stimulateur & {$[8,9]$} \\
\hline Glucokinase & Foie de rat & Stimulateur & [10] \\
\hline Albumine & Foie de rat & Stimulateur & [11] \\
\hline Amylase & Pancréas de rat & Stimulateur & [12] \\
\hline Caséine & $\begin{array}{l}\text { Explants de glandes } \\
\text { mammaires de rat }\end{array}$ & Stimulateur & [13] \\
\hline $\begin{array}{l}\text { Glycérol-3-phosphate } \\
\text { déshydrogénase }\end{array}$ & $\begin{array}{l}\text { Préadipocytes Ob17 et } \\
\text { 3T3-F442A }\end{array}$ & Stimulateur & [14] \\
\hline $\begin{array}{l}\text { Glycéraldéhyde-3- } \\
\text { phosphate déshydro- } \\
\text { génase }\end{array}$ & $\begin{array}{l}\text { Préadipocytes 3T3- } \\
\text { F442A }\end{array}$ & Stimulateur & [15] \\
\hline
\end{tabular}

culier grâce à l'utilisation d'animaux diabétiques traités par l'insuline, sont généralement d'une interprétation délicate puisque, lors du traitement hormonal substitutif, la concentration insulinique n'est pas l'unique paramètre variable. En revanche, dans ce type d'étude, la culture cellulaire offre l'avantage d'un contrôle plus strict de l'environnement hormonal, même si l'utilisation de certains modèles cellulaires peut conduire à des résultats quelquefois très différents, voire contradictoires, de ceux obtenus in vivo.

Les études réalisées à la fois chez l'animal et grâce à des cultures de cellules répondant à des concentrations physiologiques en insuline, comme les hepatocytes en culture primaire, les cellules d'hépatome de rat ou les préadipocytes murins, ont permis récemment de préciser les diverses $m / s n^{\circ} 2$ vol. 4 , férier 88 gènes, même și les connaissances sont encore bien moins étendues que pour le mode d'action d'autres effecteurs, quelques travaux réalisés ces dernieres années montrent que l'hormone peut modifier l'expression d'un gène au niveau aussi bien transcriptionnel que post-transcriptionnel.

\section{Effets transcriptionnels}

Le premier effet transcriptionnel de l'insuline a été rapporté en 1984 par le laboratoire de D.K. Granner (Vanderbilt University, Nashville, USA) lors de l'étude de la régulation hormonale de l'expression du gène de la phosphoénolpyruvate carboxykinase (PEPCK) dans des cellules d'hépatome de rat. L'expression de la PEPCK, enzyme limitante de la néoglucogenèse hépatique, est dépendante de stimuli nutritionnels et hormonaux. Lors du jeûne, le contenu hépatique en PEPCK augmente fortement; la réalimentation par un régime riche en glucides provoque un retour rapide à l'état basal. Plusieurs hormones sont impliquées dans le contrôle de la synthèse de l'enzyme dans le foie: le glucagon, l'adrénaline, la noradrénaline, l'ACTH et les glucocorticoïdes sont des effecteurs positifs alors que l'insuline exerce un puissant effet inhibiteur. La quantification des contenus hépatiques en ARN messager de la PEPCK par des techniques de traduction in vitro[18] ou par hybridation avec des sondes d'ADN complémentaires [19, 20] a montré que les variations des taux de synthèse de la protéine PEPCK sont corrélées à des variations parallèles du contenu en ARN messager, ce qui indique que la régulation de l'expression du gène se déroule à un niveau prétraductionnel.

Les cellules de la lignée H4IIE, dérivant d'un hépatome de rat et possédant plusieurs caractéristiques de cellules hépatiques différenciées, ont été utilisees par le laboratoire de D.K. Granner comme modèle d'étude de ce contrôle multihormonal de l'expression du gène de la PEPCK. 


\section{RÉFÉRENCES}

3. Avruch J, Alexander MC, Palmer JL, et al. Role of insulin-stimulated protein phosphorylation in insulin action. Fed Proc 1982; 41 : 2629-33.

4. Beale EG, Andreone $\mathrm{T}$, Koch $\mathrm{S}$, et al. Insulin and glucagon regulate cytosolic phosphoenolpyruvate carboxykinase (GTP) mRNA in rat liver. Diabetes 1984 ; 33 : 32832.

5. Granner DK, Andreone TL. Insulin modulation of gene expression. Diabetes Metab Rev 1985; 1 : 1-32.

6. Dani C, Doglio A, Grimaldi P, Ailhaud G. Expression of the phosphoenolpyruvate carboxykinase gene and its insulin regulation during differentiation of preadipose cell lines. Biochem Biophys Res Commun 1986 138: 468-75.

7. Pry TA, Porter TA. Control of fatty acid synthetase mRNA levels in rat liver by insulin, glucagon, and dibutyryl cyclic AMP. Biochem Biophys Res Commun 1981 ; 100 : 1002 9.

8. Munnich A, Marie J, Reach G, Vaulon S Simon MP, Kahn A. In vivo hormonal control of L-type pyruvate kinase gene expression. J Biol Chem 1984; 259 : 10228-31.

9. Noguchi $T$, Inoue $H$, Tanaka T. Transcriptional and post-transcriptional regulation of L-type pyruvate kinase in diabetic rat liver by insulin and dietary fructose. $J$ Biol Chem 1986; 260 : 14393-7.

10. Spence JT. Levels of translatable mRNA coding for rat liver glucokinase. $J$ Biol Chem 1983 ; 258 : 9143-6.

11. Peavy DE, Taylor JM, Jefferson LS. Correlation of albumin production rates and albumin mRNA levels in liver of normal, diabetic, and insulin-treated diabetic rats. Proc Natl Acad Sci USA 1978; 75 : 5879.83.

12. Korc M, Owerback D, Zuinto C, et al. Pancreatic islet-acinar interaction: amylase messenger RNA levels are determined by insulin. Science 1981 ; 213 : 351-3.

13. Bolander Jr FF, Nicholas KR, Van Wyk $\mathrm{JJ}$, et al. Insulin is essential for accumulation of casein mRNA in mouse mammary epithelial cells. Proc Natl Acad Sci USA 1981; 78 : 5682-4.

14. Dani C, Grimaldi P, Ailhaud G. Insulin regulation of glycerol-3-phosphate dehydrogenase gene expression in $\mathrm{Obl} 7$ adipose cells. In : Belfrage $P$, Donner $J$, Stralfors $P$, eds. Mechanisms of Insulin Action. Amsterdam : Elsevier, 1986 : 383-94.

15. Alexander M, Curtis G, Avruch J, et al. Insulin regulation of protein biosynthesis in differential 3T3 adipocytes: regulation of glyceraldehyde-3-phosphate dehydrogenase. $J$
Comme les hépatocytes in vivo, les cellules H4IIE répondent aux traitements par l'AMP cyclique ou par des analogues non métabolisés - et par la dexaméthasone par une augmentation rapide du contenu en ARN messager PEPCK. L'addition d'insuline au milieu de culture diminue les niveaux de messager PEPCK et inhibe totalement les effets des agents inducteurs AMP cyclique ou dexaméthasone. Des expériences de transcription in vitro sur noyaux isolés (test de run-on) ont montré que les variations des contenus en ARN messager PEPCK dans les cellules H4IIE traitées par les effecteurs positifs ou négatifs sont dues à des modifications des taux de transcription du gène. L'effet répresseur de l'insuline sur l'activité transcriptionnelle du gène de la PEPCK se produit à des concentrations très faibles en hormone (concentration de demi-effet de 10 picomolaire), et est un effet rapide (demi-effet atteint en 15 minutes) et indépendant de la synthèse protéique. De plus, il est important de noter que l'action de l'insuline sur la transcription du gène PEPCK est dominante sur celle des effecteurs positifs, puisque le traitement des cellules par l'insuline empêche ou inhibe totalement les effets de l'AMP cyclique et/ou de la dexaméthasone. En outre, en l'absence de tout inducteur, l'insuline réprime fortement l'expression basale du gène PEPCK dans les cellules d'hépatome [5].

Depuis la mise en évidence de l'effet de l'insuline sur la transcription du gène de la PEPCK dans les cellules d'hépatome, d'autres exemples de contrôles transcriptionnels par l'insuline ont été décrits pour d'autres gènes. Ainsi l'insuline exerce sur l'expression du gène albumine dans les cellules H4IIE [21] et du gène de l'adipsine -gène exprimant une protéase à sérine sécrétée dont le rôle au cours de la différenciation adipocytaire est encore mal connu [22]- des effets répresseurs très semblables à ceux exercés par l'hormone sur le gène
PEPCK dans les cellules d'hépatome. Outre ces effets répresseurs, l'insuline peut également exercer des effets stimulateurs sur le taux de transcription de certains gènes. Dans les lignées préadipocytaires de souris, les taux de transcription des gènes de la glycérol-3phosphate déshydrogénase (GPDH) [14] et de la glycéraldéhyde-3-phosphate déshydrogénase (GAPDH) [15] sont augmentés par des concentrations physiologiques en insuline. Des effets permissifs de l'insuline ont de plus été mis en évidence pour l'activité transcriptionnelle des gènes de l'ovalbumine dans l'oviducte de poule [23] et de la caséine dans la glande mammaire de rat [24] en association avec les estrogènes et la prolactine, respectivement.

Chez le rat diabétique, l'administration d'insuline provoque au niveau hépatique, une augmentation importante du taux de transcription du gène de la pyruvate kinase $[8,9]$. Cet effet stimulateur de l'hormone paraît mettre en jeu des mécanismes différents de ceux impliqués dans l'effet répresseur de l'hormone sur l'expression du gène de la PEPCK dans les cellules H4IIE. Ainsi, dans le cas du gène de la pyruvate kinase, l'effet de l'insuline nécessite pendant plusieurs heures une synthèse protéique active et n'est pas dominant par rapport à celui de l'AMP cyclique, ici effecteur négatif.

A l'heure actuelle, les recherches s'orientent vers la découverte des événements qui, succédant à l'interaction de l'hormone avec son récepteur membranaire, vont répercuter l'information au niveau nucléaire et moduler le taux de transcription des gènes. Chez les eucaryotes, le contrôle par certaines hormones de la transcription des gènes de classe II $^{*}$ met en jeu l'interaction de facteurs spécifiques appelés facteurs actifs en trans avec des séquences particulières situées le plus souvent en amont et voisines

\footnotetext{
* Gène de classe II: transcrits par l'ARN polymérase II, spécifique des gènes codant pour les $A R N$ messagers.
} 
du site d'initiation de la transcription ${ }^{* *}$. Ainsi des séquences régulatrices très homologues d'un gène à l'autre ont pu être identifiées au voisinage de plusieurs gènes contrôlés par les stéroïdes et par l'AMP cyclique.

Dans la mesure où la plupart des gènes contrôlés par l'insuline sont également soumis à un contrôle transcriptionnel opposé par l'AMP cyclique, l'une des hypothèses pourrait être que le nucléotide cyclique joue un rôle central dans le contrôle de la transcription et que les effets insuliniques passent, en fait, via l'activation des phosphodiestérases, par une diminution de la concentration en AMP cyclique. Des expériences récentes conduisent au rejet de cette hypothèse pour la régulation de l'expression du gène PEPCK dans les cellules H4IIE puisqu'il a été montré que l'effet répresseur de l'insuline sur le taux de transcription du gène se produit en l'absence de toute modification notable du contenu cellulaire en AMP cyclique [25]. Certains auteurs ont suggéré que les effets de l'insuline sur T'expression des gènes -dont ceux de la pyruvate kinase ou de la glucokinase- seraient en fait le reflet de l'action stimulatrice de l'hormone sur le métabolisme glucidique. Cette hypothèse, dans laquelle certains métabolites spécifiques de la voie glycolytique joueraient un rôle clé au niveau transcriptionnel, n'a pu être réellement étudiée à ce jour ni in vivo, puisque l'administration d'insuline chez l'animal doit se faire en présence de glucides, ni in vitro, du fait de la faible viabilité de certaines cellules - en particulier les cellules hépatomateuses - lorqu'elles sont incubées dans des milieux pauvres ou dépourvus de sucres. L'utilisation de modèles cellulaires moins sensibles à la privation en glucides pourrait, dans un avenir proche, permettre de préciser les rôles

\footnotetext{
* Voir mini-synthèses $\mathrm{m} / \mathrm{s} n^{\circ} 7$, vol. 3 (p.428), no8, vol.3 (p.487) et $n^{\circ} 9$, vol. 3 (p. 546).

$\mathrm{m} / \mathrm{s} n^{\circ} 2$ vol. 4, feurier 88
}

respectifs des métabolites du glucose et de l'insuline sur la régulation de l'expression de certains gènes.

Les efforts se portent actuellement sur l'identification d'un élément régulateur impliqué dans les effets transcriptionnels de l'insuline. Deux équipes se sont très récemment intéressées à la mise en évidence dans le gène PEPCK d'un tel élément régulateur par l'utilisation de la technique de transfection de cellules par un plasmide contenant un gène marqueur greffé à une région génomique supposée contenir l'élément régulateur recherché. Obtenus par des approches différentes - transfection stable [26] ou transfection transitoire [27] les résultats des deux groupes sont contradictoires puisque le premier conclut à l'absence, en amont du site d'initiation de la transcription du gène de la PEPCK, d'un élément régulateur répresseur [26] alors que le deuxième apporte des arguments en faveur de la présence d'un tel élément régulateur dans un fragment génomique de $2,1 \mathrm{~kb}$ situé en amont du gène [27]. La divergence totale des résultats de ces deux expérimentations, dont les conclusions doivent être considérées avec prudence compte tenu des artéfacts toujours possibles avec ce genre de technique, rend nécessaire de nouveaux travaux qui sont réalisés en ce moment dans plusieurs laboratoires sur les gènes PEPCK et adipsine dont l'expression est réprimée par l'insuline, et aussi sur le gène GAPDH dont la transcription est stimulée par l'hormone. L'identification de ces séquences régulatrices devrait par la suite ouvrir la voie à la recherche des facteurs diffusibles actifs en trans et des événements intermédiaires impliqués dans les effets transcriptionnels de l'insuline.

\section{Effets post-transcriptionnels}

Outre ses effets au niveau transcriptionnel, l'insuline peut également moduler l'expression de cer- tains gènes en agissant sur le métabolisme post-transcriptionnel des ARN messagers correspondants. Deux exemples d'une telle action de l'insuline ont été décrits au cours de l'année 1986. Le premier exemple est celui du contrôle de l'expression du gène de la GPDH dans les lignées préadipocytaires de souris 3T3. F442A et Obl7. Dans les deux lignées, l'exposition des cellules à des concentrations physiologiques en insuline conduit à une accumulation de l'ARN messager GPDH. L'augmentation du taux de transcription du gène GPDH, mise en évidence par des expériences de run-on sur noyaux isolés, ne pourrait rendre compte à elle seule de l'effet stimulateur de l'hormone sur le contenu cellulaire en ARN messager; l'éventualité d'un effet post-transcriptionnel de l'hormone a été envisagée. La mesure des temps de demi-vie des messagers cytoplasmiques GPDH dans les cellules exposées ou non à l'hormone a révélé que le traitement par l'insuline conduit à une stabilisation des ARN messagers cytoplasmiques GPDH. De plus, le dosage des formes moléculaires nucléaires de ce messager montre que l'effet stabilisateur de l'insuline pourrait se produire très précocément au niveau des transcrits primaires ou des intermédiaires d'épissage [14].

Le deuxième exemple d'effet posttranscriptionnel a été décrit pour le gène de l'albumine dans les cellules H4IIE. Dans ces cellules l'insuline provoque une diminution rapide de la concentration en ARN messager de l'albumine, diminution due en partie à un effet transcriptionnel et surtout à un effet post-transcriptionnel non encore caractérisé au niveau moléculaire [21].

Il faut noter que cette démonstration d'un effet inhibiteur de l'insuline sur l'expression du gène de l'albumine dans les cellules H4IIE contredit les observations antérieures d'un effet positif de l'hormone sur l'expression du même gène dans les hépatocytes [11]. Cette contradiction démon- 


\section{RÉFÉRENCES}

16. Ringold GM. Steroid hormone regulation of gene expression. Ann Rev Pharmacol Toxicol 1985 ; 25 : 529-66.

17. Guyette WA, Matusik RJ, Rosen JM. Prolactin-mediated transcriptional and posttranscriptional control of casein gene expression. Cell 1979; 17 : 1013-23.

18. Kioussis D, Reshef L, Cohen H, et al. Alterations in translatable messenger RNA coding for phosphoenolpyruvate carboxykinase (GTP) in rat liver cytosol during deinduction. J Biol Chem 1978; 253 : 4327-32.

19. Yoo-Warren H, Cimbala MA, Felz K, Monahan JE, Leis JP, Hanson RW. Identification of a DNA clone to phosphoenolpyruvate carboxykinase (GTP) from rat cytosol. $J$ Biol Chem 1981 ; 256 : 10224-7.

20. Beale EG, Hartley JL, Granner DK. $\mathrm{N}^{6}, \mathrm{O}^{2}$-dibutyryl cyclic AMP and glucose regulate the amount of messenger RNA coding for hepatic phosphoenolpyruvate carboxykinase (GTP). J Biol Chem 1982; 257 : 2022-82.

21. Straus DS, Takemoto CD. Insulin negatively regulates albumin $\mathrm{mRNA}$ at the transcriptional and post-transcriptional level in rat hepatoma cells. J Biol Chem 1987; 262 : 1955-60.

22. Cook KS, Groves DL, Min YM, et al. A developmentally regulated mRNA from 3T3 adipocytes encodes a novel serine protease homologue. Proc Natl Acad Sci USA 1985 ; $82: 6480-4$.

23. Evans MI, McKnight GS. Regulation of the ovalbumin gene: Effects of insulin, adenosine 3',5'-monophosphate and estrogen. Endocrinology 1984 ; 115 : 368-77.

24. Chomczymski P, Qasba P, Topper YJ. Essential role of insulin in transcription of the rat 25000 molecular weight casein gene. Science 1984 ; 226 : 1326-8.

25. Beale EG, Koch SR, Brotherton AFA, et al. Insulin decreases H4IIE cell PEPCK mRNA by a mechanism that does not involve cAMP. Diabetes $1986 ; 35: 546-9$.

26. Wynsham-Boris A, Short JM, Loose DS, et al. Characterization of the phosphoenolpyruvate carboxykinase (GTP) promoter-regulatory region. J Biol Chem 1986; 261 : 971420.

27. Magnusson MA, Quinn PG, Granner DK. Multihormonal regulation of PEPCK-CAT fusion genes: insulin's effects oppose to those of cAMP and dexamethasone. J Biol Chem 1987 ; 262 : 14917-20.

28. Shaw G, Kamen R. A conserved AU sequence from the 3'-untranslated region of GM-GSF mRNA mediates selective messen-

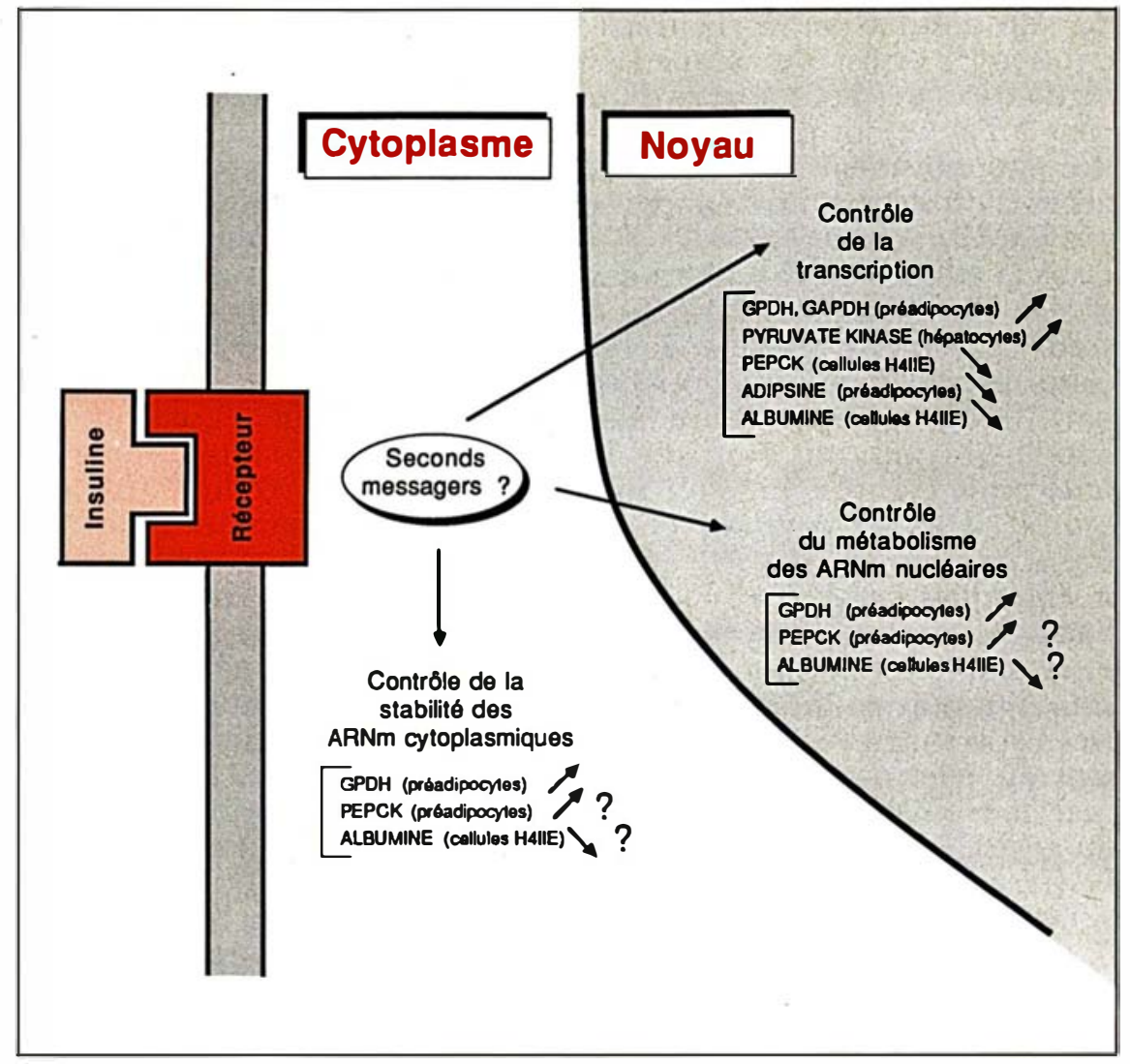

Figure 1. Cibles des effets de l'insuline sur l'expression des gènes. GPDH = glycérol-3-phosphate déshydrogénase ; PEPCK = phosphoénolpyruvate carboxykinases; GAPDH = glycéraldéhyde 3 phosphate déshydrogénase; H4IIE = lignée des cellules.

tre que si les modèles cellulaires sont nécessaires à l'étude des événements moléculaires de l'action hormonale, ils ne peuvent constituer qu'une étape préalable aux études réalisées in vivo.

Le contrôle de l'expression de ces deux gènes par l'insuline, qui affecte à la fois la synthèse et la dégradation des ARN messagers, est semblable à celle décrite pour la régulation par la prolactine de l'expression du gène de la caséine dans les cellules de la glande mammaire [17].

Des résultats récents de notre groupe indiquent que l'insuline pourrait également moduler l'expression de certains gènes en agissant uniquement au niveau posttranscriptionnel. Ceci pourrait être le cas pour l'expression du gène de la PEPCK dans les cellules préadipocytaires. Dans les préadipocytes, l'insuline exerce un puissant effet stimulateur sur la concentration en ARN messager PEPCK [6], sans affecter le taux de transcription du gène, ce qui suggère que le traitement de ces cellules par l'insuline conduit à une modification sélective de la maturation des transcrits primaires ou du temps de demi-vie des ARN messagers PEPCK.

Quels sont les mécanismes moléculaires impliqués dans ces régulations par l'insuline du métabolisme de certains AR N messagers? Un modèle dans lequel la modulation du temps de dmi-vie des ARN messagers ferait intervenir la reconnaissance d'une séquence spécifique non codante par des facteurs dont la nature chimique et/ou la concentration intracellulaire seraient soumises à un contrôle par l'hormone peut être proposé. Cette hypothèse de travail est confortée 
par la récente mise en évidence de l'existence dans certains ARN messagers - dont la stabilité est transitoirement modulable, tels les ARN codant pour les protooncogènes myc, fos et myb ou pour certaines lymphokines - de séquences spécifiques importantes pour le degré de stabilité du messager dans les cellules [28]. L'identification de séquences régulatrices dans les $A R N$ messagers dont le métabolisme est soumis à une régulation par l'insuline permettrait, dans un avenir plus lointain, la caractérisation des facteurs qui interagissent avec ces éléments régulateurs.

En conclusion, ces dernières années ont amené la démonstration de l'importance jouée par la modulation de l'expression des gènes par l'insuline dans la régulation de plusieurs voies métaboliques. Les mécanismes d'action de l'insuline sont multiples puisque l'hormone peut affecter, positivement ou négativement, à la fois la synthèse et le métabolisme post-transcriptionnel des ARN messagers codés par certains gènes. Ces effets sont complexes puisque l'hormone peut affecter de manière opposée l'expression de plusieurs gènes dans la même cellule (PEPCK et pyruvate kinase dans l'hépatocyte ou adipsine/GPDH dans l'adipocyte) ou l'expression du même gène dans les cellules différentes (PEPCK dans l'hépatocyte et dans les préadipocytes) (figure 1). Cette complexité des effets de l'insuline est probablement le reflet de la multiplicité des voies métaboliques contrôlées et du grand nombre de tissus cibles de l'hormone.

La description de divers modèles expérimentaux devrait permettre dans un avenir relativement proche l'identification des séquences impliquées dans les effets transcriptionnels et posttranscriptionnels de l'insuline et donc ouvrir la voie aux recherches sur les seconds messagers qui participent à la transmission du message hormonal de la membrane plasmique jusqu'au gène et à l'ARN messager

\section{Summary}

In recent years, numerous examples of insulin modulation of specific gene expression have been described by in vivo and in vitro studies. The diversity of the functions of the proteins encoded by insulin-regulated mRNAs, and the variety of tissues affected, suggest that there is a major mechanism by which insulin regulates the amount of protein and physiological and metabolic processes. Depending on the gene or the tissue studied, various mechanisms are involved in insulin regulation of gene expression. The hormone exerts inhibitory and stimulatory effects on the transcription rate of various genes in the same cell and, in addition to these transcriptional effects, insulin action on gene expression may involve some posttranscriptional events, including cytoplasmic mRNA stabilization. At the moment, the molecular events implicated in insulin effects on synthesis and/or degradation of specific mRNAs are not elucidated, but the active investigations in this field can lead in the near future to the identification of nucleotidic sequences and regulatory factors involved in these similar genre regulations.

\section{TIRÉS A PART}

P. Grimaldi : laboratoire de biologie du déve loppement du tissu adipeux, centre de biochimie (Cnrs LP 7300), faculté des Sciences, parc Valrose, 06034 Nice Cedex, France. 